\title{
OBJECT METAMORPHOSIS BASED ON GENERALIZED MORPHOLOGY TRANSFORMATION
}

\author{
Hua Li, Wenyu Liu, Guangxi Zhu, Yaoting Zhu \\ Huazhong University of Science \& Technology. \\ Wuhan Hubei, P. R. China
}

\begin{abstract}
A metamorphosis or a morphing is the process of continuously transforming one object into another, and are popular in computer animation, industrial design, and growth simulation. In this paper, a novel approach is presented for computing continuous shape transformation between polyhedral objects in this paper. Metamorphosis can be achieved by decomposing two objects into sets of individual convex sub-objects respectively and constructing the mapping between two sets, this method can solve the metamorphosis problem of two non-homotopic objects (including concave objects and holey objects). The results of object metamorphosis are discussed in this paper. The experiments show that this method can generate natural, high quality metamorphosis results with simple computation. This method can also be used in font composition and interpolation between two keyframes in $2 \mathrm{D}$ and $3 \mathrm{D}$ computer animation automatically.
\end{abstract}

\section{Introduction}

The method used to describe nonrigid body motion and metamorphosis is becoming more and more important in science simulation, virtual reality, animal motion, human face muscle motion, modeloriented image coding and so on. It gives the animator the ability to "fill" an animation between keyframed objects by inbetweening. It allows the designer to blend existing shapes in order to create new shapes. In recent years, many algorithms have been proposed to compute a transformation between two shape models.

Research effort in the metamorphosis field initially focused on the 2D problem. For image space techniques, Beier and Neely [1] presented an elegant feature-based approach based on fields of influence surrounding 2D control primitives. A treatise of image warping methods can be found in Wolberg's [2] book. As for 2D polygonal shapes, Sederberg and Greenwood [3] proposed a solution to the vertex correspondence problem, and the vertex path problem was dealt with in Sederberg et al. [4]. Other 2D algorithms that deal with the vertex path of polygonal shapes were proposed by Shapira and Rappoport [5], and by Goldstein and Gotsman [6].

Some 3D metamorphosis algorithms deal with sampled or volumetric representation of the objects. Hughes [7] proposed transforming the object into the Fourier domain, and then interpolating the low frequencies over time while the high frequencies are slowly added in, thus minimizing the object distortion caused by its high-frequency components. A method using the wavelet transform instead of the Fourier transform was proposed by He et al. [8]. Lerios et al. [9] extended the work of Beier and Neely [1] where fields of influence of 3D primitives were used to warp a voxel space. Kaul and Rossignac [10] computed the Minkowski sum of scaled versions of the models, and then used it to obtain a smooth 
transition between the models. Galin and Akkouche[11] have presented an algorithm for blob metamorphosis based on Minkowski sums. Payne and Toga [12] gave a distance-field volumetric representation of 3D biomedical objects. However, no warping or correspondence was established between the source and the target object. This lack of control over the morphing process often yields poor results. Cohen-Or [13] presented a method that uses the distance-field volumetric representation of 3D objects, established a point-to-point warp function between the two models that can be seen as a way of enforcing topological correspondence and geometrical properties.

Other 3D metamorphosis algorithms that apply to objects represented by their boundaries. Chen and Parent [14] briefly addressed an extension of a transformation for piecewise linear 2D contours to 3D objects represented by a set of planar contours. Bethel and Uselton[15] add degenerate vertices and faces to two polyhedra until they have a common vertex neighborhood graph. Kent [16] have presented a shape transformation algorithm for genus-zero polyhedral that involves projecting the models onto a sphere. Kanai et al. [17], as well as Bao and Peng [18], have presented algorithms for shape transformation of genus-zero polyhedra using harmonic maps. Lazarus and Verroust [19] have proposed a method based on skeletal curves. Shapiro and Tai [20] propose a polyhedron realization algorithm for shape transformation. Parent [21] has presented an approach that splits the surface of the model into pairs of sheets of faces and recursively subdivides them until they have the same topology. DeCarlo and Gallier [22] have proposed a morphing technique that establishes correspondence by allowing the user to divide the surface into triangular and quadrilateral patches that can be projected onto a plane. Gregory [23] proposed a approach shares their theme, but they improve upon several restrictions in DeCorlo's technique, making it easier for the user to specify correspondence between complicated models. Sun et al. [24] presented a solution to the vertex path problem of polyhedral models, which is an extension of the intrinsic shape transformation scheme suggested in Sederberg et al. [4] for 2D polygons. The reader is referred to Lazarus [25] for more survey about metamorphosis.

Ghosh first introduced morphology to the representation of 2D polygons and applied it to the research of regular polyhedron modeling [26, 27]. He also proposed a fast algorithm of Minkowski addition of 2D polygons [28,29]. Mount and Silverman proposed the morphologically decomposable concept of polygons. They also proved that convex polyhedrons can be decomposed to the Minkowski addition of several indecomposable parts in $O\left(n^{3}\right)$ times [30]. Kaul and Rossignac introduced morphology to $3 \mathrm{D}$ object metamorphosis [10], they defined the intermediate polyhedral objects using linear interpolation based on Minkowski sums of the two original polyhedra. The faces of the deformed shapes have a constant orientation and their vertices move on a straight line between a vertex of the initial shape and a vertex of the final shape. Moreover, when the two original shapes are convex, these faces from the boundary of the deformed polyhedron. A related work [31] uses the Bézier formulation with the Minkowski sum to compute each intermediate shape as a point in the space of polyhedra. This extension adds flexibility to the technique and is very intuitive for convex solids. The work of Metamorphosis based on Morphology also includes Galin and Akkouche [11]. These morphological methods is very effective for convex polyhedron metamorphosis, but if the object is a concave polyhedron or a polyhedron with holes, this method will lead to morbid results in which one cannot obtain the expected metamorphosis object (See Figure1.). The reason is that Minkowski addition is an expanding operator. During the process of computing the concave polyhedron's Minkowski addition with another polyhedron, it is possible that the concave polyhedron deforms to a convex polyhedron first and then deforms to a concave polyhedron again, all of which will lead to a morbid result. 


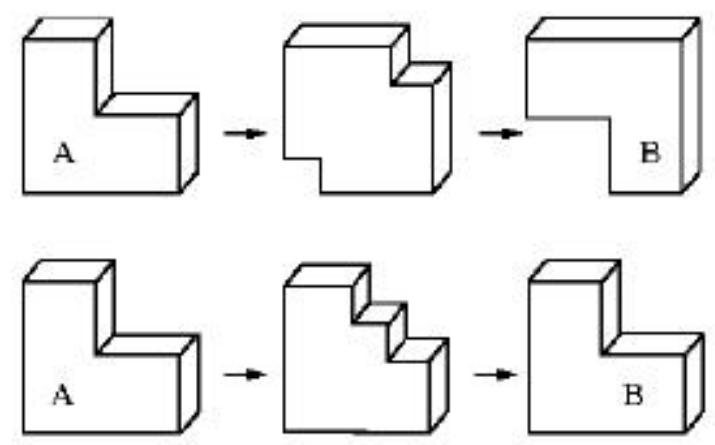

Figure 1: Intermediate shapes $(A \oplus B) / 2$ using the Minkowski sum [10]

In order to solve the morbid result of nonrigid body (especially the concave body) metamorphosis based on morphology, we proposed a novel method to first decompose objects into a series of convex subsets, then set up the mapping of two convex subsets of objects, and then compute the Minkowski addition of two convex subsets according to their mapping relationship. The final step is to combine them to obtain the final result. This novel method is a generalized optimized nonlinear interpolation method, which has no restriction on objects and can achieve ideal continuous interpolation with high speed and high quality. It can also be expanded to the interpolation of 3D solids.

\section{The Principle of Object Metamorphosis}

\subsection{CONVEX DECOMPOSITION}

The first step to decompose concave polygons to convex polygons is triangulation. In order to achieve uniform metamorphosis, each decomposed triangle should both share the same vertices and as few as possible. After decomposition, merge the two borders upon the convex polygon (a triangle polygon is a convex polygon) to a new convex polygon until any two borders upon the convex polygons cannot be merged again.

Suppose $A$ includes $K$ disjunct polygons (a polygon is called a connected weight) $D_{k}^{\prime}, 1 k K$. Further suppose $B$ includes $L$ disjunct polygons (a polygon is called a connected weight) $D_{l}^{\prime \prime}, 1 \quad l \quad L$. Decompose $A$ to $m$ convex polygons, $A_{1}, A_{2}, A_{m}$. Any two of them have no intersection. Decompose $B$ to $n$ convex polygons, $B_{1}, B_{2}$, $B_{n}$. Any two of them have no intersection. $A_{i}$ is called the convex subset of $A$, and $B_{j}$ is called the convex subset of $B$.

We construct the minimum rectangles which encompass polygon $A$ or $B$, and set up coordinate systems that the coordinate origin superpose with the left-down vertex of the minimum rectangle, respectively. Then, if we link the origin and the barycenter of convex subset $A_{i}$, we obtain a vector $\vec{r}_{A_{i}}$, which is called the coordinate vector of the convex subset $A_{i}$. Similarly, when we link the origin and the barycenter of convex subset $B_{j}$, we also obtain a vector $\vec{r}_{B_{j}}$, which is called the coordinate vector of the convex subset $B_{j}$. Then, there is one coordinate vector in each convex subset. Convenient for comparing different polygons, the maximum border length of the minimum rectangle should be turned over to 1 . 


\subsection{MAPPING [32-34]}

The matching of $A$ and $B$ includes two steps because there is not only one polygon in $A$ and $B$ (see Figure2 Chinese "three").

\subsubsection{Mapping the connected weights}

First matching the connected weight (a polygon is called a connected weight), there are $K$ connected weights in $A$, and $L$ connected weights in $B$. Then each connected weight in $A$ matches with a connected weight in $B$, and each connected weight in $B$ matches with a connected weight in $A$ according to the optimization matching principle. Thus, the mapping of convex subsets is limited in only two matching connected weights. Suppose a connected weight $D_{k}^{\prime}$ in $A$ which matches with a connected weight $D_{l}^{\prime \prime}$ in $B$, namely, then $D_{l}^{\prime \prime}$ is formed from $D_{k}^{\prime}$ metamorphosis. The method of relax iteration matching is selected to obtain connected weight matching optimization.

Suppose $h$ denotes the convex package of a connected weight. The minimum and maximum coordinate $x$ of $A$ are $x_{1}^{\prime}, x_{2}^{\prime}$ respectively; the minimum and maximum coordinate $y$ of $A$ are $y_{1}^{\prime}, y_{2}^{\prime}$; the minimum and maximum coordinate $x$ of $B$ are $x_{1}^{\prime \prime}, x_{2}^{\prime \prime}$; and the minimum and maximum coordinate $y$ of $B$ are $y_{1}^{\prime \prime}, y_{2}^{\prime \prime}$. The attribute of a convex package is defined as a 3-tuple $(p, s, t)$, where $p$ is the barycenter's coordinate $(x, y)$ of a convex package, $s$ is the area of a convex package, and $t$ is the correlation function of max, min $x, y$ coordinate of two convex packages.

Suppose convex packages $h_{1}=\left(p_{1}, s_{1}, t_{1}\right) \quad h_{2}=\left(p_{2}, s_{2}, t_{2}\right)$. Then the similitude function of $h_{1}, h_{2}$ is defined as $m\left(h_{1}, h_{2}\right), 0 \quad m\left(h_{1}, h_{2}\right) \quad 1$ and

$$
m\left(h_{1}, h_{2}\right)=w_{1} v_{1}+w_{2} v_{2}+w_{3} v_{3}
$$

where $w_{i}$ is the weight coefficient $\sum w_{i}=1$.

$$
\begin{gathered}
\text { Define } \quad v_{1}=\frac{\left(x_{1}-x_{2}\right)^{2}+\left(y_{1}-y_{2}\right)^{2}}{\left(x_{1}+y_{1}\right)^{2}+\left(x_{2}+y_{2}\right)^{2}} \quad\left(x_{1}, y_{1}\right),\left(x_{2}, y_{2}\right) \text { are barycenters of } h_{1}, h_{2} \\
v_{2}=\frac{\left|s_{1}-s_{2}\right|}{\max \left(s_{1}-s_{2}\right)} \quad \text { where } s_{1}, s_{2} \text { are areas of } h_{1}, h_{2} \\
v_{3}=t=1-d_{1} d_{2} d_{3} d_{4}
\end{gathered}
$$

If $x, y$ in convex package $h_{1}, x \in\left[x_{1}^{\prime}, x_{2}^{\prime}\right], y \in\left[y_{1}^{\prime}, y_{2}^{\prime}\right]$

If $x, y$ in convex package $h_{2}, x \in\left[x_{1}^{\prime \prime}, x_{2}^{\prime \prime}\right], y \in\left[y_{1}^{\prime \prime}, y_{2}^{\prime \prime}\right]$

$$
d_{1}=\left|x_{1}^{\prime}-x_{1}^{\prime \prime}\right|, d_{2}=\left|x_{2}^{\prime}-x_{2}^{\prime \prime}\right|, d_{3}=\left|y_{1}^{\prime}-y_{1}^{\prime \prime}\right|, d_{4}=\left|y_{2}^{\prime}-y_{2}^{\prime \prime}\right|
$$

If $h_{1}$ matches with $h_{2}$, at least two $d_{i}(1 \leq i \leq 4)$ should get small values near to the smallest, because $0 \leq d_{i} \leq 1$, so $v_{3}$ has the largest value.

There are $K$ connected weights in $A$, and $L$ connected weights in $B$. Constitute similitude matrix $S_{K \times L}(i, j$, num $)$ by $m\left(h_{1}, h_{2}\right)$. Where num is the iteration number, iteration is carried out according to Formula (1): 


$$
S_{K \times L}(i, j, \text { num })=\sum_{h \neq i}\left\{\max _{r \neq j} S_{K \times L}(h, r, \text { num }-1)\right\} \times S_{K \times L}(i, j, n u m-1) /(K-1)
$$

For each $D_{k}^{\prime}$ in $A$, only match with one $D_{l}^{\prime \prime}$ in $B$, if $S_{K \times L}\left(k, l^{\prime}\right.$, num $)$ is less than some constant $T$, set $S_{K \times L}(k, l '$, num $)=0$, where $l^{\prime} \neq l$. Constitute similitude matrix $S_{L \times K}(j, i, n u m)$, and carry out the relax iteration according to Formula(2):

$$
S_{L \times K}(j, i, \text { num })=\sum_{h \neq j}\left\{\max _{r \neq i} S_{L \times K}(h, r, n u m-1)\right\} \times S_{L \times K}(j, i, n u m-1) /(L-1)
$$

For each $D_{l}^{\prime \prime}$ in $B$, only match with one $D_{k}^{\prime}$ in $A$, if $S_{L \times K}\left(l, k^{\prime}, n u m\right)$ is less than some constant $T$, set $S_{L \times K}\left(l, k^{\prime}\right.$, num $)=0$, where $k^{\prime} \neq k$. So if $D_{k}^{\prime}$ matches with $D_{l}^{\prime \prime}$, the max element values in $S_{K \times L}(k, l$, num $)$ and $S_{L \times K}(l, k, n u m)$ should be larger than $T$.

\subsubsection{Mapping the Convex subsets}

After matching the connected weights, we need to map the convex subsets. The mapping of convex subsets is limited in only two matching connected weights. If convex subset $A_{i} \subseteq D_{k}^{\prime} \quad B_{j} \subseteq D_{l}^{\prime \prime}$. Search $A_{i}$ 's mapping $B_{j}$ according to the following Formula (3):

$$
\min _{\text {for all } j \text { in } D_{l}^{\prime \prime}}\left(\left|\vec{r}_{A_{i}}-\vec{r}_{B_{j}}\right|\right)
$$

Make the above formula come into existence, and denote the relationship $(i, j)$, apparently, $(i, j)=(j$, i) The mapping algorithm of convex subsets is as follows:

1. Classify $A_{i}, B_{j}$ as the matching relationship of their corresponding connected weights, with one convex subset of $A$ only mapping another of convex subset of $B$.

2. For each $A_{i}$, get a $B_{h}$ in a corresponding convex subset; make Formula (3) come into existence, and then add relationship $(i, h)$ to relationship list $R$, and marker $B_{h}$.

3. If there are some $B_{j}$ which have no marker, search a $A_{l}$ in $A$ 's mapping convex subsets and make Formula (3) come into existence. Then add relationship $(l, j)$ to relationship list $R$ where list $R$ includes all the mapping relationships of $A, B$ 's convex subsets.

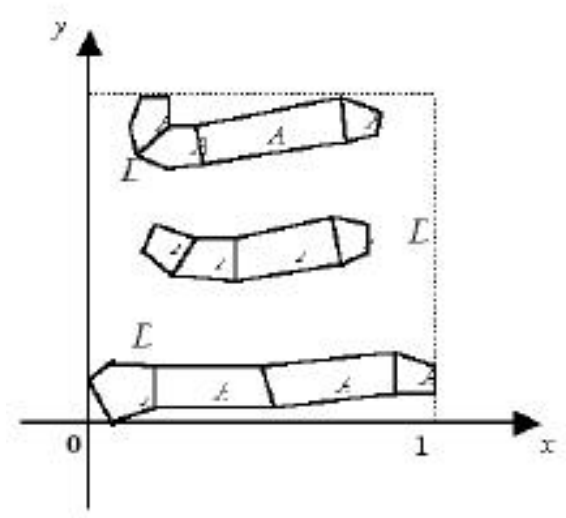

(a)

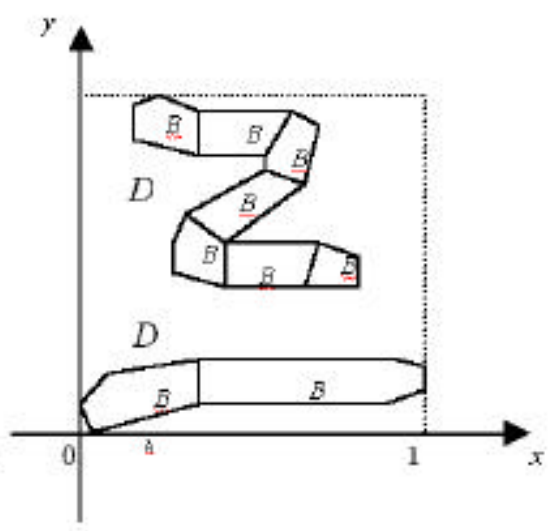

(b)

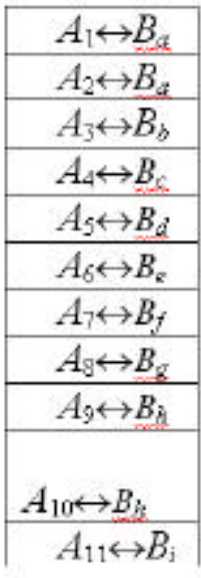

(c)

Figure 2: (a) and (b) represent two object's convex decomposition.

(c) is the mapping relationship list $R$ of convex subsets in (a) and (b). 
Figure 2 shows two Chinese script "three" $A$ and $B$. There are three connected weights $\left(D_{1}^{\prime}, D_{2}^{\prime}, D_{3}^{\prime}\right)$ and two connected weights $\left(D_{1}^{\prime \prime}, D_{2}^{\prime \prime}\right)$. $D_{1}^{\prime}$ is decomposed to 4 convex subsets, $D_{2}^{\prime}$ is decomposed to 4 convex subsets, and $D_{3}^{\prime}$ is decomposed to 4 convex subsets. $D_{1}^{\prime \prime}$ is decomposed to 7 convex subsets, and $D_{2}^{\prime \prime}$ is decomposed to 2 convex subsets. The result of connected weight matching is $D_{1}^{\prime}$ matching with $D_{1}^{\prime \prime}, D_{2}^{\prime}$ matching with $D_{1}^{\prime \prime}$, and $D_{3}^{\prime}$ matching with $D_{2}^{\prime \prime}$. The convex subset mapping is carried out according to Formula (4). The relationship list $R$ is shown in Figure2(c). The symbol $\leftrightarrow$ denotes mapping relationship.

\subsection{INTERPOLATION}

Definition 1 Suppose $A_{i}(1 \leq i \leq m), B_{j}(1 \leq j \leq n)$, and there is a mapping relationship in convex decomposing optimization mapping of $A$ and $B$, define $C^{\prime}(r)$ as the following Formula(4):

$$
C^{\prime}(r)=\underset{\text { For all i, jhaving mapping relationsh ip }}{\bigcup} \sum_{\left(r A_{i} \oplus(1-r) B_{j}\right)}
$$

$C^{\prime}(r)$ is called the metamorphosis polygon of $A, B$.

Theorem $1 C^{\prime}(1)=A \quad C^{\prime}(0)=B$ : while $A=B, C^{\prime}(r) \equiv A, 0 \leq r \leq 1$.

Proof: Because $C^{\prime}(1)=A, C^{\prime}(0)=B$, if $A=B$,

then no matter if $A$ is concave or convex, $A, B$ have the same decomposition, that is

$$
A=A_{1} \cup A_{2} \cdots \cup A_{m} \quad B=B_{1} \cup B_{2} \cdots \cup B_{m}
$$

And because $A_{i}=B_{i}(1 \leq i \leq m)$, the mapping relationship is $A_{i} \leftrightarrow B_{i}$.

If $A_{i} \bullet^{\bullet} B_{i}$ are convex polygons " as a result, there are $A_{i}=r A_{i} \oplus(1-r) B_{i}$. Then

$$
\begin{aligned}
C^{\prime}(r) & =\bigcup \sum\left(r A_{i} \oplus(1-r) B_{i}\right) \\
& =\sum \bigcup A_{i} \\
& =A
\end{aligned}
$$

Theorem $2 C^{\prime}(r) \subseteq r A \oplus(1-r) B$ exists for any $0 \leq r \leq 1$.

Proof: $r A \oplus(1-r) B=r\left(\sum_{i=1}^{m} \bigcup A_{i}\right) \oplus(1-r)\left(\sum_{j=1}^{n} \cup B_{j}\right)=\sum_{i=1}^{m} \cup r A_{i} \oplus\left(\sum_{j=1}^{n} \cup(1-r) B_{j}\right)$

$$
\supseteq \bigcup_{\text {for all i, jhaving mapping relationsh ip }}\left(r A_{i} \oplus(1-r) B_{j}\right)=C^{\prime}(r)
$$

Inference 1 Vertices of $C^{\prime}(r)$ must be on the edge or the interior of $r A \oplus(1-r) B$.

Inference 2 If $C^{\prime}(r)$ is the metamorphosis polygon when $A$ is decomposed to $m$ convex subsets, and $B$ is decomposed to $n$ convex subsets, $C^{\prime \prime}(r)$ is the metamorphosis polygon when $A$ is decomposed to $m^{\prime}$ convex subsets, and $B$ is decomposed to $n^{\prime}$ convex subsets. If $n^{\prime}>n, m^{\prime}>m$, then $C^{\prime \prime}(r) \subseteq C^{\prime}(r)$.

Definition 2 For any convex decomposition of polygons $A$ and $B, C^{\prime}(r)$ expresses their metamorphosis polygon. If a kind of convex decomposition and its metamorphosis polygon is $C_{R}(r)$, and $C_{R}(r) \subseteq C^{\prime}(r)$, then $C_{R}(r)$ is called the minimum metamorphosis polygon.

Theorem 3 If $A$ is an arbitrary polygon, and $B$ is a convex polygon, then $C^{\prime}(r) \equiv r A \oplus(1-r) B$. 
Proof: Convex decompose polygon $A$, and obtain $A \equiv \sum_{i=1}^{m} \bigcup A_{i}$, where $A_{i}$ is convex subset. Keep polygon $B$ without convex decomposition, and then the mapping relationship of $A, B$ 's convex subsets is $A_{i} \leftrightarrow B$

$C^{\prime}(r)=\bigcup \sum\left(r A_{i} \oplus(1-r) B\right)=\sum\left(\bigcup r A_{i} \oplus(1-r) B\right)=\left(\sum \bigcup A_{i}\right) \oplus(1-r) B=r A \oplus(1-r) B$

Theorem 3 shows that, if either $A$ or $B$ is convex, there is no need to convex decompose the polygons. The metamorphosis polygon will achieve the same computation result as that using a morphology operator directly. However, if you want to achieve a more natural effect, $B$ should be decomposed to several convex subsets. When the number of $B$ 's convex subsets is the same as $A$ 's, the metamorphosis can achieve the best effect.

$C_{R}(r)$ preferably describes the metamorphosis sequence of polygons $A, B$ while $r$ varies from 1 to 0 . The convex decomposition number of $A, B$ are limited so there exists the minimum metamorphosis polygon $C_{R}(r)$ of $A, B$. Normally, $C_{R}(r)$ corresponds to the triangle decomposition of $A, B$. Generally, $C^{\prime}(r)$ has the same topo-structure as $C_{R}(r)$, and $C_{R}(r) \subseteq C^{\prime}(r)$. The difference between $C^{\prime}(r)$ and $C_{R}(r)$ is the number of concave vertices; $C_{R}(r)$ has more concave vertices than $C^{\prime}(r)$, but the difference is not distinct. To improve computation speed and get a more natural metamorphosis result, we should use the maximum of $C^{\prime}(r)$, which means the number of decomposition $m, n$ should be the minimum.

$C^{\prime}(r)$ includes the coordinate translation and scaling transformation of $B$ to $A$, but it cannot describe the rotating transformation of $B$ to $A$. If $B$ 's rotating angle to $A$ is $\theta$, denote the rotating transformation matrix $R(\theta)$. Then, $R(-\theta) B$ represents rotating $-\theta$ for $B$. The generalized morphinterpolation of $A, B$ can be decomposed to the metamorphosis and rotation transform of polygons and represented in the following Formula (5):

$$
C_{\text {int }}^{\prime}(r)=R((1-r) \theta)\left(\bigcup \sum_{\text {for all i,j having mapping relationship }} r A_{i} \oplus(1-r) R(-\theta) B_{j}\right)
$$

$C_{\text {int }}^{\prime}$ is called as the generalized morph-interpolation of $A$ and $B$, simply called interpolation, $r \in[0,1]$.

\section{Experimental results and Analysis}

Figure 3 below is the generalized morph-interpolation result of a Chinese standard bold font (means "shape") and a manuscript Chinese font (means "shape"). The standard bold font is decomposed to 32 convex polygons, and the manuscript font is decomposed to 104 convex polygons. The interpolation result is very natural. The information of Chinese font peak and the little holes are also kept without any loss. The method can be applied to new Chinese or English font combination. Figure 4 is the metamorphosis sequence of a convex polyhedron with a hole to a goblet. The polyhedron is decomposed to 4 convex parts, and the goblet is decomposed to three parts: up, middle, and down. Figure 5 is another metamorphosis sequence of a 3D duck to an iron. Comparing the result with that of Cohen-Or, we can see it is satisfied. It shows that the methods proposed in this paper also can be used for 3D objects. 


\section{形形形形形形形形;}

Figure 3: Generalized morph-interpolation result of Chinese font, the middle 6 fonts are the result of generalized morph-interpolation, while $r=6 / 7,5 / 7,4 / 7,3 / 7,2 / 7,1 / 7$ respectively.

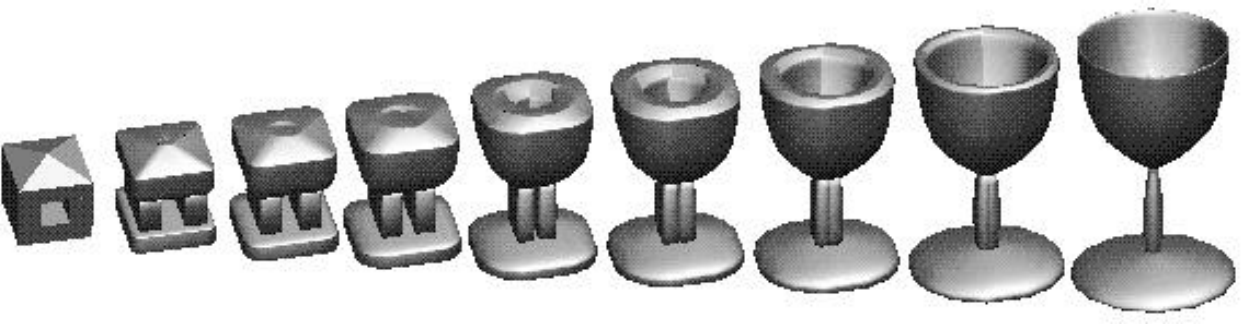

Figure 4: Metamorphosis sequence of a convex polyhedron with a hole to a goblet, the middle 7 polyhedrons are the result of generalized morph-interpolation, while $r=7 / 8,6 / 8,5 / 8,4 / 8,3 / 8,2 / 8,1 / 8$, respectively.

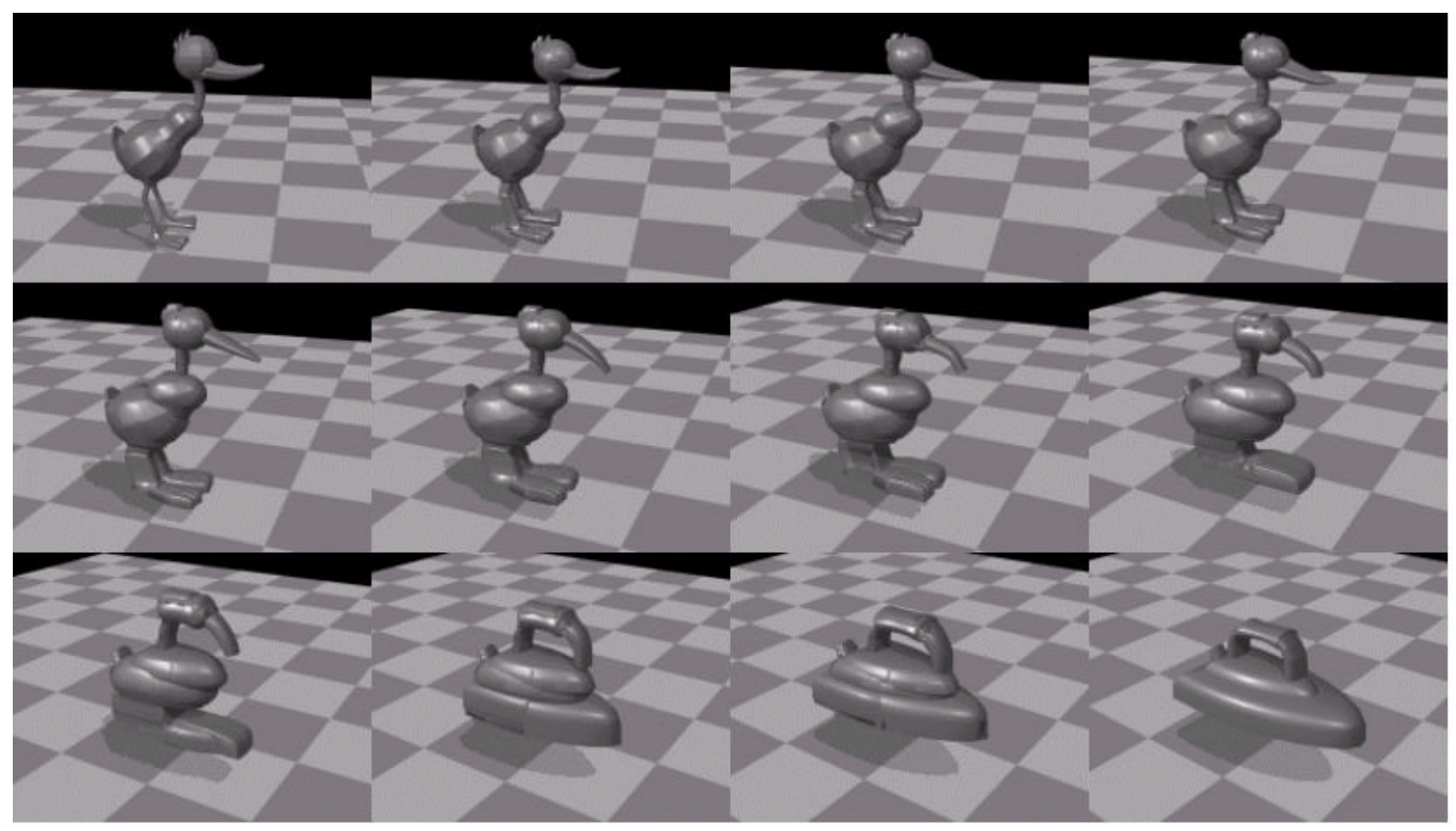

Figure 5:Metamorphosis sequence of a duck to an iron, the middle 10 polyhedrons are the result of generalized morph-interpolation, while $r=9 / 10,8 / 10,7 / 10,6 / 10,5 / 10,4 / 10,3 / 10,2 / 10,1 / 10$ respectively. 
The generalized interpolation method provides a preferable description of nonrigid objects' metamorphosis and solves the morbid problems of non-homotopic objects based on traditional morphologic methods. It can be applied widely in keyframes interpolation of 2D animation, combination of Chinese or English fonts, skeleton movement of objects, and model-oriented image coding. This method can also be simply expanded to solve the movement and metamorphosis of 3D nonrigid objects.

\section{Acknowledgment}

The project is supported by the National Natural Science Foundation of China (No.69973018).

\section{REFERENCES}

[1] T.Beier and S.Neely, "Feature-based image metamorphosis", Computer Graphics (SIGGRAPH'92), Vol.26, No.2, 1992, pp. 35-42

[2] G.Wolberg, Digital Image Warpping. IEEE Computer Society Press, Los Alamitos, Calif, 1990.

[3] T.W.Sderberg and E.Greenwood. "A physically based approach to 2D shape blending". in Proceedings of SIGGRAPH'92, Vol.26, No.2, 1992, pp. 25-34

[4] T.W.Sederberg, P.Gao, G.Wang, and H.Mu. "2D shape blending: An intrinsic solution to the vertex path problem". in proceedings of SIGGRAPH'93, Vol.27, 1993, pp. 15-18

[5] M.Shapira and A.Rappoport. "Shape blending using the star-skeleton representation". IEEE Compute, Graphics and application. Vol.15, No.3, 1995, pp. 44-50

[6] E.Goldstein and C.Gotsman. "Polygon morphing using a multiresolution representation". In Proceedings of Graphics interface'95, 1995, pp. 247-254

[7] J.F.Hughes. "Scheduled Fourier Volume Morphing”. Proc. SIGGRAPH'92, Vol.26, 1992, pp. 4346

[8] T.He, S.Wang, and A.Kaufman. "Wavelet-based volume morphing". In proceedings of Visualization'94, 1994, Octuber, pp. 85-91

[9] A.Lerios, C.D.Garfinkle, and M.Levoy, "Feature-based Volume Metamorphosis", SIGGRAPH, 1995, Vol. 29, pp. 449-464

[10] Anil Kaul and Jarek Rssignac. "Solid-Interpolation Deformations: Construction and Animation of PIPS”. Computer \& Graphics, Vol.16, No.1, 1991, pp. 107-115

[11] E.Galin and S.Akkouche. "Blob metamorphosis based on Minkowski sums". In eurographics'96, 1996, C149-C153

[12] B.A.Payne and A.W.Toga. "Distance field manipulation of surface models". IEEE CG\&A, Vol.12, No.1, 1992, pp. 65-71

[13] D.Cohen-or and D.Levin, "A.Solomovoci. Three-dimensional distance field metamorphosis". ACM Transactions on Graphics, Vol.17, No.2, 1998, pp. 116-141 
[14] S.Chen and R.E.Parent. "Shape Averaging and Its Application to Industrial Design". IEEE CG\&A, Vol.9, No.1, 1989, pp. 47-54

[15] E.W.Bethel and S.P.Uselton. "Shape distortion in computer-assisted keyframe animation", in computer animation'89, state-of-the-art in computer animation, computer graphics international, 1989, pp. $215-224$

[16] J.R.Kent, W.E.Carlson, and R.E.Parent. "Shape transformation for polyhedral objects". Computer Graphics, 1992, Vol.26, No.2, 1992, pp. 47-54

[17] T.Kanai, H.Suzuki, and F.Kimura, "3D Geometric Metamorphosis based on Harmonic Map", The Visual Computer, Vol.14, No.7, 1998, pp. 166-176

[18] Bao H, Peng Q, “Interactive 3D Morphing”, Computer Graphics Forum, Vol.17, 1998, pp. C23$\mathrm{C} 30$

[19] F.Lazarus and A.Verroust. "Metamorphosis of cylinder-like objects". The Journal of Visualization and Computer Animation, Vol.8, 1997, pp. 131-146

[20] Avner Shapiro and Ayellet Tal, "Polyhedron Realization for Shape Transformation", The Visual Computer, Vol.14, No.8/9, 1998, pp. 429-444

[21] R.E.Parent. "Shape transformation by boundary representation interpolation: a recursive approach to establishing face correspondence". Journal of Visualization Computer \& Animation, Vol. 3, 1992, pp. 219-239

[22] D.DeCarlo, J.Gallier. Topological evolution of surfaces. Graphics Interface'96, 1996, 194-203.

[23] Arthur Gregory, Andrei State, Ming C. Lin. et al. "Interactive Surface Decomposition for Polyhedral Morphing”, The Visual Computer, Vol.15, 1999, pp. 453-470

[24] Y.M.Sun, W.Wang and F.Y.Chin. "Interpolating polyhedral models using intrinsic shape parameters". in proceedings of the third pacific conference on computer graphics and applications, Pacific graphics'95, 1995, pp. 133-147

[25] Francis Lazarus and Anne Verroust, "Three-Dimensional Metamorphosis: A Survey", The Visual Computer, Vol.14, No. 8/9, 1998, pp. 373-389

[26] P.K.Ghosh. "A Solution of Polygon Containment, Spatial Planning, and Other Related Problems using Minkowski Operations". Computer Vision, Graphics, and Image Processing, Vol.49, No.1, 1990, pp. 1-35

[27] P.K.Ghosh. “A Unified Computational Framework for Minkowski Operations”. Computer \& Graphics, Vol.77, No.4, 1993, pp. 357-378

[28] P.K.Ghosh. "An Algebra of Polygons through The Notion of Negative Shapes". CVGIP, 1991, Vol.54, No.1, 1991, pp. 119-144

[29] P. K.Ghosh and P. K.Jain. “An Algebra of Geometric Shapes”. IEEE CG\&A, Vol.9, No.1, 1993, pp. $50-58$ 
[30] D.Mount and R.Silverman. "Combinatorial and Computational Aspects of Minkowski Decompositions”. Contemporary Mathematics, Vol.11, No.9, 1991, pp. 107-124

[31] J.Rossignac and A.Kaul, "AGRELS and BIBs: metamrphosis as a Bézier curve in the space of plyuhedra”, Eurographics’94, Oslo, Sep. 1994, pp:C-179-C-184

[32] LIU Wen-Yu, WAN Fei, ZHU Guang-Xi. “A Method of Modeling Based On Morphology”. IEEE International Conference on Intelligent Processing System, Beijing, 1997: 994-998

[33] LiuWenYu, LiHua, and ZhuGuangXi, "Approach to Nonrigid Body Metamorphosis Using Generalized Morph-translation", Computer Aided Drafting, Design and Manufacturing, China, Vol.10, No.1, 2000, pp. 1-8

[34] LiuWenYu, LiHua, and ZhuGuangXi, "A Novel Method For Description Nonrigid Body Motion And Its Applications Based On Generalized Morpho-interpolation”, Journal of Software/RuanJian XueBao, to be published.

\section{AUTHOR CONTACT INFORMATION}

Hua Li

Department of Electronics \& Information Engineering

State Education Commission Laboratory for Image Processing and Intelligent Control

Huazhong University of Science \& Technology

Wuhan Hubei 430074

P. R. China

Email link: mailto:lihua 2001@mails1.hust.edu.cn

Wenyu Liu

Department of Electronics \& Information Engineering

State Education Commission Laboratory for Image Processing and Intelligent Control

Huazhong University of Science \& Technology

Wuhan Hubei 430074

P. R. China

Email link: mailto:liuwy@mail.hust.edu.cn

Guangxi Zhu

Department of Electronics \& Information Engineering

State Education Commission Laboratory for Image Processing and Intelligent Control

Huazhong University of Science \& Technology

Wuhan Hubei 430074

P. R. China

Yaoting Zhu

Department of Electronics \& Information Engineering

State Education Commission Laboratory for Image Processing and Intelligent Control

Huazhong University of Science \& Technology

Wuhan Hubei 430074

P. R. China 